\title{
Extremal solutions for measure differential inclusions via Stieltjes derivatives
}

\author{
Giselle A. Monteiro ${ }^{1}$ and Bianca Satco ${ }^{2 *}$ (10
}

\author{
"Correspondence: \\ bianca.satco@eed.usv.ro \\ ${ }^{2}$ Faculty of Electrical Engineering \\ and Computer Science; Integrated \\ Center for Research, Development \\ and Innovation in Advanced \\ Materials, Nanotechnologies, and \\ Distributed Systems for Fabrication \\ and Control (MANSiD), Stefan cel \\ Mare University of Suceava, \\ Suceava, Romania \\ Full list of author information is \\ available at the end of the article
}

\begin{abstract}
Stieltjes derivatives represent a new unification of discrete and continuous calculus consisting in a differentiation process with respect to a given non-decreasing function $g$. This notion infers a new class of differential equations which has shown to have many applications. Herein we explore the use of such derivatives in the study of multivalued equations, the so-called $g$-differential inclusions. Such multivalued differential problems simply consist in replacing the usual derivatives by Stieltjes derivatives (also known as g-derivatives). Using Baire category methods, we investigate extremal solutions for $g$-differential inclusions. It is shown that $g$-differential inclusions offer an alternative approach to measure-driven problems; therefore, the existence of extremal solutions for measure differential inclusions is obtained as a simple consequence of the results for this new type of inclusions.
\end{abstract}

MSC: 34A60; 34A36; 26A24; 26A45; 46G05; 49K21

Keywords: Measure differential inclusion; Extremal solution; Stieltjes derivative

\section{Introduction}

Studying phenomena involving mixed discrete-continuous behavior (so-called hybrid systems) is, in general, a difficult task. When discrete perturbations occur on a finite set of moments, the theory of impulsive differential equations offers the necessary tools, but for dealing with infinitely many abrupt changes more refined methods are needed. Some possible approaches to address such problems rely on generalized differential equations [21, $26,28]$, measure differential equations $[10,17,25]$, and the analysis on time scale domains $[5,13]$. Remarkably, under certain assumptions, these approaches happen to be equivalent (see $[17,18,30])$.

When dealing with problems in control theory, economics, or game theory, one has to consider set-valued functions; and consequently, the models may involve multivalued differential equations (i.e., differential inclusions, [2]). In the recent development of the theory of differential inclusions, the study of measure differential inclusions has gained popularity as it includes as special cases differential and difference inclusions, impulsive and hybrid problems (cf. [11-13, 16, 27, 29, 31]).

In [24], the notion of derivative with respect to monotone functions $g$ has been revitalized. Earlier studies on such a kind of derivatives and its connection with Stieltjes integrals include Young [34], Daniell [14], Ward [33] and, more recently, Bendová and Malý [4]. With the latest attention brought to the study of Stieltjes differentiability in [24], a new

(c) The Author(s) 2019. This article is distributed under the terms of the Creative Commons Attribution 4.0 International License (http://creativecommons.org/licenses/by/4.0/), which permits unrestricted use, distribution, and reproduction in any medium, provided you give appropriate credit to the original author(s) and the source, provide a link to the Creative Commons license, and indicate if changes were made. 
class of differential problems has gained popularity: the so-called $g$-differential equations [20]. These equations can be regarded as the Stieltjes-differential counterpart of measure differential equations, and they have shown to be widely applicable to solving practical problems (e.g., [22, 23]).

In the present paper, we extend the notion of $g$-differential equation to the set-valued case by introducing a class of differential inclusions based on the notion of Stieltjes derivative. In other words, we consider inclusions in which the usual derivative is replaced by a derivative with respect to a monotone function $g$; therefore called $g$-differential inclusions. In some sense, this type of inclusions encompasses measure-driven inclusions, offering a new approach to impulsive multivalued problems.

Our aim is to establish the existence of extremal solutions for $g$-differential inclusions, i.e., inclusions of the form

$$
\begin{aligned}
& x_{g}^{\prime}(t) \in F(t, x(t)) \quad \mu_{g} \text {-a.e. } \\
& x(0)=0,
\end{aligned}
$$

where $x_{g}^{\prime}$ stands for the derivative with respect to a given non-decreasing function $g$. Herein, we follow the approach to Stieltjes derivatives found in [20, 24]; while this derivative seems fairly suitable for our purposes, we acknowledge that other definitions available in the literature may allow for a study within more general classes of functions, e.g., [4, $33]$. Like in $[7,8,15]$, which are concerned with the particular case of classical differential inclusions, in the present work we use methods from the Baire category theory. More precisely, for $w:[0,1] \rightarrow \mathbb{R}^{n}$, we consider an auxiliary problem

$$
\begin{aligned}
& x_{g}^{\prime}(t) \in F^{w(t)}(t, x(t)) \quad \mu_{g} \text {-a.e. } \\
& x(0)=0
\end{aligned}
$$

where $F^{w}(t, x)$ denotes the subset of $F(t, x)$ which maximizes the inner product with $w$, that is,

$$
F^{w}(t, x)=\left\{y \in F(t, x) ;\langle y, w\rangle=\max _{z \in F(t, x)}\langle z, w\rangle\right\} .
$$

We prove that the set of all continuous functions $w:[0,1] \rightarrow \mathbb{R}^{n}$ for which the solutions of the $g$-differential problem above satisfy also the extremal problem

$$
\begin{aligned}
& x_{g}^{\prime}(t) \in \operatorname{ext} F(t, x(t)) \quad \mu_{g} \text {-a.e. } \\
& x(0)=0
\end{aligned}
$$

is residual in $C\left([0,1], \mathbb{R}^{n}\right)$ (Theorem 14$)$. As a consequence, we obtain the existence of extremal solutions for $g$-differential inclusions, wherefrom we deduce an analogue theorem for measure-driven inclusions.

Besides the clear importance of the knowledge on extremal solutions in optimization theory and in relaxation problems, it is worth mentioning that our result can be seen as the first existence theorem for measure differential inclusions with non-convex right-hand side. Namely, we obtain a generalization of Filippov's classical result contained in [19]. 


\section{Preliminary results. Integrals and derivatives}

Throughout this paper, $\mathcal{P}_{\mathrm{ck}}\left(\mathbb{R}^{n}\right)$ stands for the family of non-empty convex compact subsets of $\mathbb{R}^{n}$. By $\mu_{g}$ we denote the Lebesgue-Stieltjes measure generated by a non-decreasing left-continuous function $g$.

The results and proofs in this paper rely deeply on the theories of Lebesgue and Lebesgue-Stieltjes integral. We fix the notation

$$
\int_{[0, t)} f(s) d \mu \text { and } \int_{[0, t)} f(s) d g(s)
$$

respectively for the Lebesgue integral of $f$ with respect to a Borel measure $\mu$, and for the Lebesgue-Stieltjes integral of $f$ with respect to a monotone function $g$. Recalling that every finite Borel measure in $\mathbb{R}$ agrees with some Lebesgue-Stieltjes measure, we can find a nondecreasing left-continuous function $g: \mathbb{R} \rightarrow \mathbb{R}$ so that $\mu(B)=\mu_{g}(B)$ for every Borel set $B$ (see [9, Theorem 3.21]). Hence, the integral notions above are in some sense equivalent.

Next, following [24], we recall some basic definitions and properties of Stieltjes derivatives (see also [25]).

Definition 1 Let $g:[0,1] \rightarrow \mathbb{R}$ be a non-decreasing left-continuous function. The derivative with respect to $g$ (or the $g$-derivative) of a function $f:[0,1] \rightarrow \mathbb{R}^{n}$ at a point $t \in[0,1]$ is given by

$$
\begin{aligned}
& f_{g}^{\prime}(t)=\lim _{s \rightarrow t} \frac{f(s)-f(t)}{g(s)-g(t)} \quad \text { if } g \text { is continuous at } t, \\
& f_{g}^{\prime}(t)=\lim _{s \rightarrow t^{+}} \frac{f(s)-f(t)}{g(s)-g(t)} \quad \text { if } g \text { is discontinuous at } t
\end{aligned}
$$

provided the limit exists.

It is worth mentioning that if $t$ is a point of discontinuity of $g$, the $g$-derivative $f_{g}^{\prime}(t)$ exists if and only if the sided limit $f(t+)$ exists, and in this case

$$
f_{g}^{\prime}(t)=\frac{f(t+)-f(t)}{g(t+)-g(t)}
$$

Note that Definition 1 has no meaning in the parts of the domain in which $g$ is constant; denote such a region by $C_{g}$. However, as observed in [24], this is not a big loss because $\mu_{g}\left(C_{g}\right)=0$.

Fundamental theorems of calculus are essential when taking into account the connection between integrals and derivatives. Such a result has been proven in [33] for PerronStieltjes integral, while for Lebesgue-Stieltjes integrals it reads as follows [24, Theorem 2.4].

Theorem 2 Let $g:[0,1] \rightarrow \mathbb{R}$ be a non-decreasing left-continuous function. Iff $:[0,1] \rightarrow$ $\mathbb{R}^{n}$ is Lebesgue-Stieltjes integrable with respect to $g$ and

$$
F(t)=\int_{[0, t)} f(s) d g(s), \quad t \in[0,1]
$$

then $F_{g}^{\prime}=f$ on $[0,1] \backslash N$, where $N \subset[0,1]$ and $\mu_{g}(N)=0$. 
Related to the result above, we have the following notion of absolute continuity which traces back to the work of Ward in [33].

Definition 3 Let $g:[0,1] \rightarrow \mathbb{R}$ be a non-decreasing left-continuous function. A function $f:[0,1] \rightarrow \mathbb{R}^{n}$ is absolutely continuous with respect to $g$ (or $g$-absolutely continuous) if for every $\varepsilon>0$ there exists $\delta>0$ such that

$$
\sum_{j=1}^{m}\left\|f\left(b_{j}\right)-f\left(a_{j}\right)\right\|<\varepsilon
$$

for any family $\left\{\left(a_{j}, b_{j}\right)\right\}$ of disjoint subintervals of $[0,1]$ satisfying

$$
\sum_{j=1}^{m}\left(g\left(b_{j}\right)-g\left(a_{j}\right)\right)<\delta
$$

By $\mathcal{A C}_{g}\left([0,1], \mathbb{R}^{n}\right)$ we denote the set of all $g$-absolutely continuous functions.

Notably, a function in $\mathcal{A C}_{g}\left([0,1], \mathbb{R}^{n}\right)$ shares some properties with $g$, namely: it is leftcontinuous on $(0,1]$, it is continuous at the points where $g$ is continuous, and it is constant in the intervals where $g$ is constant (cf. [24, Proposition 5.3]). Moreover, every $g$-absolutely continuous function is of bounded variation, therefore also regulated (i.e., it has only discontinuities of first kind).

As one could expect, the function $F$ from Theorem 2 is $g$-absolutely continuous [33, Theorem 12]. The following result, a second fundamental theorem of calculus for Lebesgue-Stieltjes, has been proven in [24, Theorem 5.4] (a version involving a more general notion of integral can be found in [33, Theorem 13]).

Theorem 4 Let $g:[0,1] \rightarrow \mathbb{R}$ be a non-decreasing left-continuous function. If $F:[0,1] \rightarrow$ $\mathbb{R}^{n}$ is g-absolutely continuous, then $F_{g}^{\prime}$ exists $\mu_{g}$-a.e., and

$$
F(t)=F(0)+\int_{[0, t)} F_{g}^{\prime}(s) d g(s) \quad \text { for every } t \in[0,1]
$$

A particular case of $g$-absolute continuity is a consequence of a Lipschitz-type property that we define in the sequel.

Definition 5 Let $g:[0,1] \rightarrow \mathbb{R}$ be a non-decreasing left-continuous function. Then $f:$ $[0,1] \rightarrow \mathbb{R}^{n}$ is said to be $g$-Lipschitz with (Lipschitz) constant $M>0$ if

$$
\left\|f(t)-f\left(t^{\prime}\right)\right\| \leq M\left(g(t)-g\left(t^{\prime}\right)\right), \quad \text { whenever } 0 \leq t^{\prime}<t \leq 1 .
$$

The next result describes a class of functions which are $g$-Lipschitz.

Lemma 6 Let $g:[0,1] \rightarrow \mathbb{R}$ be a non-decreasing left-continuous function, and let $f:$ $[0,1] \rightarrow \mathbb{R}^{n}$ be a $\mu_{g}$-measurable function with $\|f(t)\| \leq M$ on $[0,1]$ for some constant $M>0$. Then the function $F:[0,1] \rightarrow \mathbb{R}^{n}$ defined by

$$
F(t)=\int_{[0, t)} f(s) d g(s), \quad t \in[0,1]
$$

is g-Lipschitz with constant $M$. 
Proof Indeed, the following inequality

$$
\left\|F(t)-F\left(t^{\prime}\right)\right\|=\left\|\int_{\left[t^{\prime}, t\right)} f(s) d g(s)\right\| \leq M \int_{\left[t^{\prime}, t\right)} d g(s)=M\left(g(t)-g\left(t^{\prime}\right)\right)
$$

holds for every choice of $0 \leq t^{\prime}<t \leq 1$.

We remark that every $g$-absolute continuous function $f$ is bounded and $g$-continuous; in other words, for each $t \in[0,1], f$ satisfies the following: given $\varepsilon>0$ there exists $\delta>0$ such that

$$
|g(s)-g(t)|<\delta \quad \Rightarrow \quad\|f(s)-f(t)\|<\varepsilon .
$$

(It is worth highlighting that the notion of continuity with respect to a function also appears in [33] with a slightly different connotation.)

The space of $g$-continuous functions, $\mathcal{B C}_{g}\left([0,1], \mathbb{R}^{n}\right)$, endowed with the supremum norm is a Banach space (see [20] for details). Next, we recall a useful criterion for compactness presented in [20, Proposition 5.6].

Theorem 7 Let $\mathcal{S} \subset \mathcal{A C}_{g}\left([0,1], \mathbb{R}^{n}\right)$ be such that $\{x(0): x \in \mathcal{S}\}$ is bounded. If there exists $h:[0,1] \rightarrow \mathbb{R}$ Lebesgue-Stieltjes integrable with respect to $g$ such that

$$
\left\|x_{g}^{\prime}(t)\right\| \leq h(t) \quad \text { for } \mu_{g} \text {-a.e. } t \in[0,1] \text { and for all } x \in \mathcal{S}
$$

then $\mathcal{S}$ is relatively compact in $\mathcal{B C}_{g}\left([0,1], \mathbb{R}^{n}\right)$.

\section{Main results}

The notion of derivative with respect to monotone functions infers a new class of differential equations [20]. Along these lines, one can also consider a more general class of differential inclusions:

$$
\begin{aligned}
& x_{g}^{\prime}(t) \in F(t, x(t)) \quad \mu_{g} \text {-a.e. } \\
& x(0)=0,
\end{aligned}
$$

where $F:[0,1] \times \mathbb{R}^{n} \rightarrow \mathcal{P}_{\text {ck }}\left(\mathbb{R}^{n}\right)$ is a multifunction and $g:[0,1] \rightarrow \mathbb{R}$ is a non-decreasing left-continuous function. Differential inclusions of the form (2) will be called $g$-differential inclusions, where the derivative $x_{g}^{\prime}$ is understood in the sense of Definition 1. Clearly, in the case when $g$ is the identity function, problem (2) corresponds to a classical differential inclusion (see [2, 32]).

A solution of problem (2) is defined in a natural way as follows.

Definition 8 A function $x \in \mathcal{A C}_{g}\left([0,1], \mathbb{R}^{n}\right)$ is a solution of problem (2) if there exists $N \subset[0,1]$, with $\mu_{g}(N)=0$, such that the $g$-derivative of $x$ satisfies

$$
x_{g}^{\prime}(t) \in F(t, x(t)) \quad \text { for every } t \in[0,1] \backslash N
$$

and $x(0)=0$. 
Note that, by considering derivatives with respect to non-decreasing functions $g$, although the inclusion in (2) is not meaningful at the points of the set $C_{g}$, we can still infer some property about the solution. Indeed, the $g$-absolute continuity ensures that the solution remains constant in the intervals where $g$ is constant (cf. [24, Proposition 5.3]). Moreover, recalling the expression of the $g$-derivative at discontinuity points (1), it becomes clear in which manner the jumps of a solution of (2) are controlled by the set-valued function $F$.

Remark 9 By Theorem 4, a solution $x \in \mathcal{A C}_{g}\left([0,1], \mathbb{R}^{n}\right)$ of (2) satisfies

$$
x(t)=\int_{[0, t)} x_{g}^{\prime}(s) d g(s), \quad t \in[0,1]
$$

Thus, if $F$ is a bounded multifunction, it follows from Lemma 6 that $x$ is $g$-Lipschitz continuous.

To motivate the study of problem (2), consider a measure differential inclusion

$$
d x(t) \in F(t, x(t)) d \mu, \quad x(0)=0,
$$

where $\mu$ is a finite regular Borel measure. According to [11], a function $x:[0,1] \rightarrow \mathbb{R}^{n}$ is a solution of (3) if there exists a $\mu$-integrable function $f:[0,1] \rightarrow \mathbb{R}^{n}$ such that

$$
x(t)=\int_{[0, t)} f(s) d \mu, \quad t \in[0,1], \quad \text { and } \quad f(t) \in F(t, x(t)) \quad \mu \text {-a.e. }
$$

Since the integral above can be understood as a Lebesgue-Stieltjes integral with respect to some non-decreasing left-continuous function $g$, that is,

$$
x(t)=\int_{[0, t)} f(s) d g(s), \quad t \in[0,1]
$$

it follows from Theorem 2 and (4) that $x_{g}^{\prime}(t)=f(t) \in F(t, x(t)) \mu_{g}$-a.e. Besides, as observed in Sect. 2, the function $x$ is $g$-absolutely continuous; in other words, a solution $x$ of (3) is a solution of problem (2) for some choice of $g$.

On the other hand, if $x$ is a solution of (2), then $x$ is $g$-absolutely continuous and Theorem 4 ensures that

$$
x(t)=\int_{[0, t)} x_{g}^{\prime}(s) d g(s), \quad t \in[0,1] .
$$

Since $x_{g}^{\prime}(t) \in F(t, x(t)) \mu_{g}$-a.e., we conclude that $x$ is the solution of measure differential inclusion

$$
d x(t) \in F(t, x(t)) d \mu_{g}, \quad x(0)=0 .
$$

This indicates a correspondence between $g$-differential inclusions and measure differential inclusions. 
Theorem 10 Let $F:[0,1] \times \mathbb{R}^{n} \rightarrow \mathcal{P}_{\mathrm{ck}}\left(\mathbb{R}^{n}\right)$. The following assertions hold:

1. Given a finite regular Borel measure $\mu$, if $x:[0,1] \rightarrow \mathbb{R}^{n}$ is a solution of the measure differential inclusion (3), then $x$ is a solution of a g-differential inclusion (2) for some function $g:[0,1] \rightarrow \mathbb{R}$ such that $\mu=\mu_{g}$.

2. Given a non-decreasing left-continuous function $g:[0,1] \rightarrow \mathbb{R}$, if $x:[0,1] \rightarrow \mathbb{R}^{n}$ is a solution of the $g$-differential inclusion (2), then $x$ is a solution of a measure differential inclusion (3) with $\mu=\mu_{g}$.

Impulsive differential inclusions represent another important class of problems which pertains to $g$-differential inclusions. Indeed, let us consider an impulsive problem with multivalued jumps $I_{k}: \mathbb{R}^{n} \rightarrow \mathcal{P}_{\mathrm{ck}}\left(\mathbb{R}^{n}\right), k=1, \ldots, m$, described by the following differential inclusion:

$$
\begin{aligned}
& y^{\prime}(t) \in \bar{F}(t, y(t)) \quad \text { a.e. in }[0,1] \backslash\left\{t_{k}: k=1, \ldots, m\right\}, \\
& y\left(t_{k}+\right)-y\left(t_{k}\right) \in I_{k}\left(y\left(t_{k}\right)\right), \\
& y(0)=0,
\end{aligned}
$$

where $\bar{F}:[0,1] \times \mathbb{R}^{n} \rightarrow \mathcal{P}_{\text {ck }}\left(\mathbb{R}^{n}\right), 0<t_{1}<\cdots<t_{m}<1$. Traditionally, the solutions $y$ are considered in the space of piecewise continuous functions, which are left-continuous and have right limit at the discontinuity points, e.g., [3].

For $t \in[0,1]$ and $y \in \mathbb{R}^{n}$, put

$$
\begin{aligned}
& g(t)=t+\sum_{k=1}^{m} \chi_{\left(t_{k}, 1\right]}(t), \\
& F(t, y)= \begin{cases}\bar{F}(t, y), & \text { if } t \in[0,1] \backslash\left\{t_{k}: k=1, \ldots, m\right\}, \\
I_{k}(y), & \text { if } t=t_{k} \text { for some } k=1, \ldots, m .\end{cases}
\end{aligned}
$$

Thus, it is not hard to see that, with this particular choice of $g$ and $F$, the impulsive problem (5) corresponds to a $g$-differential inclusion (2). Indeed, if $y$ is a solution (in the sense described above) of the impulsive problem (5), then $y_{g}^{\prime}(t)=y^{\prime}(t)$ at the points $t$ in $[0,1] \backslash\left\{t_{k}\right.$ : $k=1, \ldots, m\}$ where the usual derivative exists, while by (1) we know that

$$
y_{g}^{\prime}\left(t_{k}\right)=\frac{y\left(t_{k}+\right)-y\left(t_{k}\right)}{g\left(t_{k}+\right)-g\left(t_{k}\right)}=y\left(t_{k}+\right)-y\left(t_{k}\right) \quad \text { for } k=1, \ldots, m .
$$

In summary, $y_{g}^{\prime}(t) \in F(t, y(t))$ a.e. in $[0,1]$.

Naturally, our object of study allows for a more general formulation of impulsive problems as countably many impulse moments can be accounted. Recall that since any monotone real function has at most a countable number of discontinuity points, the LebesgueStieltjes measure $\mu_{g}$ generated by such a function $g$ can be decomposed as follows:

$$
\mu_{g}=\mu_{g}^{C}+\sum_{n=1}^{\infty}\left(g\left(t_{k}^{+}\right)-g\left(t_{k}\right)\right) \delta_{t_{k}},
$$

where $A=\left\{t_{k}, k \in \mathbb{N}\right\}$ is the set of atoms of $\mu_{g}, \delta_{t_{k}}$ stands for the Dirac measure concentrated at the point $t_{k}$, and $\mu_{g}^{C}$ is the nonatomic part of the measure $\mu_{g}$ (i.e., the Stieltjes 
measure generated by the continuous part of $g$ ). Using the definition of the $g$-derivative, problem (2) can be rewritten as follows:

$$
\begin{aligned}
& x_{g}^{\prime}(t) \in \bar{F}(t, x(t)) \quad \mu_{g}^{C} \text {-a.e. in }[0,1] \backslash A, \\
& x\left(t_{k}+\right)-x\left(t_{k}-\right) \in\left(g\left(t_{k}+\right)-g\left(t_{k}\right)\right) I_{k}\left(x\left(t_{k}\right)\right), \quad t_{k} \in A, \\
& x(0)=0,
\end{aligned}
$$

where the multifunctions $\bar{F}, I_{k}, k \in \mathbb{N}$, are such that, for $t \in[0,1]$ and $y \in \mathbb{R}^{n}$,

$$
F(t, y)= \begin{cases}\bar{F}(t, y), & \text { if } t \notin A, \\ I_{k}(y), & \text { if } t=t_{k} \in A .\end{cases}
$$

In the particular case when the continuous part of $g$ generates the Lebesgue measure, the problem above corresponds to an impulsive differential inclusion with multivalued jumps and with possibly countably many fixed impulse points. Therefore, the investigation of $g$-differential inclusions may extend what is found in the literature regarding impulsive differential inclusions $[1,3]$.

Our main goal is to discuss the question of extremal solutions for problem (2). In other words, the focus of our attention is the $g$-differential problem associated with (2)

$$
\begin{aligned}
& x_{g}^{\prime}(t) \in \operatorname{ext} F(t, x(t)) \quad \mu_{g} \text {-a.e. } \\
& x(0)=0
\end{aligned}
$$

which is to be understood as the $g$-derivative $x_{g}^{\prime}(t)$ taking values within the set of extreme points of $F(t, x(t))$. We recall that, for some convex set $C \subset \mathbb{R}^{n}$, a point $a$ is called an extreme point of $C$ if, for any $u, v \in C$, the equality $a=\frac{u+v}{2}$ implies $a=u=v$.

In what follows by $\mathcal{F}$ and $\mathcal{F}^{\text {ext }}$ we denote the solution set of (2) and (6), respectively. Given $w \in \mathbb{R}^{n}$, for $t \in[0,1]$ and $x \in \mathbb{R}^{n}$, let $F^{w}(t, x)$ be the compact convex subset of $F(t, x)$ consisting in all points maximizing the inner product with $w$ :

$$
F^{w}(t, x)=\left\{y \in F(t, x) ;\langle y, w\rangle=\max _{z \in F(t, x)}\langle z, w\rangle\right\}
$$

Under the assumption that $F$ is a bounded Pompeiu-Hausdorff continuous multifunction, for each continuous function $w:[0,1] \rightarrow \mathbb{R}^{n}$, taking

$$
F^{w}(t, x)=F^{w(t)}(t, x)
$$

we have that $F^{w}:[0,1] \times \mathbb{R}^{n} \rightarrow \mathcal{P}_{\text {ck }}\left(\mathbb{R}^{n}\right)$ defines a set-valued function which is upper semicontinuous with respect to the second variable. Thanks to the equivalence between $g$ differential inclusions and measure differential inclusions, Theorem 10, the solvability of the problem

$$
x_{g}^{\prime}(t) \in F^{w(t)}(t, x(t)) \quad \mu_{g} \text {-a.e., } \quad x(0)=0,
$$


is equivalent to the existence of solution of

$$
d x(t) \in F^{w}(t, x(t)) d \mu_{g}, \quad x(0)=0 .
$$

Noting that for $F$ bounded and continuous the multifunction $F^{w}$ satisfies the assumptions of [11, Theorem 6], problem (8) has a solution; as a consequence, we have the following result.

Theorem 11 Let $g:[0,1] \rightarrow \mathbb{R}$ be a non-decreasing left-continuous function and $F:$ $[0,1] \times \mathbb{R}^{n} \rightarrow \mathcal{P}_{\mathrm{ck}}\left(\mathbb{R}^{n}\right)$ be a bounded Pompeiu-Hausdorff continuous multifunction. Then, for every $w \in C\left([0,1], \mathbb{R}^{n}\right)$, problem (7) has at least one solution.

Let $\mathcal{F}^{w}$ denote the set of solutions of (7). We will see that the inclusion $\mathcal{F}^{w} \subset \mathcal{F}^{\text {ext }}$, $w \in C\left([0,1], \mathbb{R}^{n}\right)$, somehow defines a generic property in the space of continuous functions. In other words, for 'almost' any choice of $w \in C\left([0,1], \mathbb{R}^{n}\right)$, the solutions of (7) also satisfy inclusion (6).

One important tool in the further investigation is the so-called Choquet function ([6], p. 158) defined as follows: for $K \in \mathcal{P}_{\text {ck }}\left(\mathbb{R}^{n}\right)$ and $y \in \mathbb{R}^{n}$, if $y \in K$ put

$$
\Phi(K, y)=\sup \left\{\int_{0}^{1}\|f(\xi)-y\|^{2} d \xi ; f:[0,1] \rightarrow K, \int_{0}^{1} f(\xi) d \xi=y\right\},
$$

otherwise, we set $\Phi(K, y)=-\infty$.

Lemma 12 Let $x \in \mathcal{A C}_{g}\left([0,1], \mathbb{R}^{n}\right)$ be given. Then $x \in \mathcal{F}^{\text {ext }}$ if and only if

$$
\int_{[0,1)} \Phi\left(F(t, x(t)), x_{g}^{\prime}(t)\right) d g(t)=0
$$

Proof Recall that $\Phi(K, y)=0$ if and only if $y$ is an extreme point of the convex set $K$. Consequently, $x \in \mathcal{F}^{\text {ext }}$ if and only if $\Phi\left(F(t, x(t)), x_{g}^{\prime}(t)\right)=0 \mu_{g}$-a.e. in $[0,1]$, which is then equivalent to

$$
\int_{[0,1)} \Phi\left(F(t, x(t)), x_{g}^{\prime}(t)\right) d g(t)=0
$$

(due to the positivity of the function $\Phi$ ).

In what follows $B$ denotes the closed unit ball in $\mathbb{R}^{n}$, while $\partial B$ corresponds to its boundary.

Inspired by the methods used in [7], we will consider an auxiliary function $\phi:[0,1] \times$ $\mathbb{R}^{n} \times \partial B \rightarrow \mathbb{R}$ defined by

$$
\phi(t, x, w)=\max \left\{\Phi(F(t, x), y) ; y \in F^{w}(t, x)\right\} .
$$

Further, for $\lambda>0$, we denote

$$
\phi^{\lambda}(t, x, w)=\max \left\{\phi\left(t, x^{\prime}, w^{\prime}\right)-\lambda\left|x^{\prime}-x\right|-\lambda\left|w^{\prime}-w\right| ; x^{\prime} \in \mathbb{R}^{n}, w^{\prime} \in \partial B\right\} .
$$


In other words, for each $t \in[0,1], \phi^{\lambda}(t, \cdot, \cdot)$ corresponds to the minimum of all $\lambda$-Lipschitz continuous functions which are greater than $\phi(t, \cdot, \cdot)$.

In the next lemma we summarize some properties of these auxiliary functions $\phi$ and $\phi^{\lambda}$. Its proof follows the same arguments used by Bressan in [7], pp. 2395-2396, and we omit it.

Lemma 13 Let $F:[0,1] \times \mathbb{R}^{n} \rightarrow \mathcal{P}_{\mathrm{ck}}\left(\mathbb{R}^{n}\right)$ be a Pompeiu-Hausdorff continuous multifunction such that $F(t, x) \subset B$ for every $(t, x) \in[0,1] \times \mathbb{R}^{n}$. The functions $\phi, \phi^{\lambda}:[0,1] \times \mathbb{R}^{n} \times$ $\partial B \rightarrow \mathbb{R}, \lambda>0$, defined above satisfy:

(i) $\phi$ is upper semicontinuous.

(ii) $\phi(t, x, w)=0$ for a.e. $w \in \partial B$ and for all $x \in \mathbb{R}^{n}, t \in[0,1]$.

(iii) $\phi^{\lambda}(t, x, w) \rightarrow \phi(t, x, w)$ when $\lambda \rightarrow \infty$, pointwise in $[0,1] \times \mathbb{R}^{n} \times \partial B$.

(iv) for every $\delta>0$, there exists $\lambda>0$ such that

$$
f_{\partial B} \phi^{\lambda}(t, x, w) d w<\delta \quad \text { for every } x \in B, t \in[0,1]
$$

where we integrate with respect to the probability measure uniformly distributed over the sphere $\partial B$.

The main result of this paper is stated below.

Theorem 14 Let $g:[0,1] \rightarrow \mathbb{R}$ be a non-decreasing left-continuous function and $F:$ $[0,1] \times \mathbb{R}^{n} \rightarrow \mathcal{P}_{\mathrm{ck}}\left(\mathbb{R}^{n}\right)$ be a bounded Pompeiu-Hausdorff continuous multifunction such that

for every $w, x \in \mathbb{R}^{n}, t \in A$, the set $F^{w}(t, x)$ is a singleton,

where $A=\left\{t_{k}, k \in \mathbb{N}\right\}$ is the set of atoms of $\mu_{g}$. Then

$$
W=\left\{w \in C\left([0,1], \mathbb{R}^{n}\right) ; \mathcal{F}^{w} \subset \mathcal{F}^{\mathrm{ext}}\right\}
$$

is a residual subset of $C\left([0,1], \mathbb{R}^{n}\right)$.

Proof In the case $n=1$, note that

$$
W \supseteq\left\{w \in C([0,1], \mathbb{R}) ; w(t) \neq 0 \mu_{g} \text {-a.e. }\right\} \supset \bigcap_{k=1}^{\infty} C_{k},
$$

where for each $k \in \mathbb{N}$

$$
C_{k}:=\left\{w \in C([0,1], \mathbb{R}) ; w \equiv 0 \text { in } A_{w}, \mu_{g}\left(A_{w}\right)<1 / k, \text { where } A_{w} \subset[0,1]\right.
$$

is a finite union of intervals whose endpoints belong to $[0,1] \backslash \bar{A}\}$.

Thus, to see that $W$ is residual, it is enough to show that $C_{k}, k \in \mathbb{N}$, is a dense open subset of $C([0,1], \mathbb{R})$. 
Each $C_{k}$ is dense. Indeed, given $h \in C([0,1], \mathbb{R})$ and $\varepsilon>0$, we know that there exists a polynomial $P_{\varepsilon}$ such that

$$
\sup _{t \in[0,1]}\left\|P_{\varepsilon}(t)-h(t)\right\|<\frac{\varepsilon}{2}
$$

Let $x_{1}, \ldots, x_{p_{\varepsilon}}$ be the roots of $P_{\varepsilon}$. If $x_{\ell} \in \bar{A}$ for some $\ell$, then choose $\delta>0$ such that $x_{i}+\delta \notin \bar{A}$ for every $i=1, \ldots, p_{\varepsilon}$ and $\left|P_{\varepsilon}(t-\delta)-P_{\varepsilon}(t)\right|<\frac{\varepsilon}{2}$ for every $t \in[0,1]$. Thus the polynomial $P_{\varepsilon}^{*}(t)=P_{\varepsilon}(t-\delta)$ clearly belongs to $C_{k}$ for every $k \in \mathbb{N}$ and

$$
\sup _{t \in[0,1]}\left\|P_{\varepsilon}^{*}(t)-h(t)\right\|<\varepsilon
$$

proving that any $\varepsilon$-neighborhood of the function $h$ intersects $C_{k}, k \in \mathbb{N}$.

Each $C_{k}$ is open. Indeed, given $w \in C_{k}$, denote by $p$ the number of intervals of $A_{w}$, and let

$$
\alpha=\frac{\frac{1}{k}-\mu_{g}\left(A_{w}\right)}{2}, \quad \delta<\frac{\alpha}{2 p},
$$

where $\delta$ is chosen in such a way that there are no atoms of $\mu_{g}$ in the neighborhood of $A_{w}$ of radius $\delta$. Then any function $f \in C([0,1], \mathbb{R})$ with

$$
\sup _{t \in[0,1]}\|f(t)-w(t)\|<\delta
$$

has the property that it is null at most on the set $A_{f}$ formed by $A_{w}$ together with $2 p$ intervals of length $\frac{\alpha}{2 p}$ each. Hence, $\mu_{g}\left(A_{f}\right) \leq \mu_{g}\left(A_{w}\right)+2 p \frac{\alpha}{2 p}<\frac{1}{k}$ and $f \in C_{k}$, so the case $n=1$ is clarified.

Now assume $n \in \mathbb{N}, n \geq 2$. In view of Lemma 12 , we can write

$$
W=\left\{w \in C\left([0,1], \mathbb{R}^{n}\right) ; \int_{[0,1)} \Phi\left(F(t, x(t)), x_{g}^{\prime}(t)\right) d g(t)=0 \text { for every } x \in \mathcal{F}^{w}\right\} .
$$

Thus, to prove that $W$ is of second Baire category in $C\left([0,1], \mathbb{R}^{n}\right)$, it suffices to show that, for every $\varepsilon>0$,

$$
W^{\varepsilon}=\left\{w \in C\left([0,1], \mathbb{R}^{n}\right) ; \int_{[0,1)} \Phi\left(F(t, x(t)), x_{g}^{\prime}(t)\right) d g(t)<\varepsilon \text { for every } x \in \mathcal{F}^{w}\right\}
$$

is open and dense in $C\left([0,1], \mathbb{R}^{n}\right)$. This will be done in two steps. Without loss of generality we can assume that $F(t, x) \subset B$ for every $(t, x) \in[0,1] \times \mathbb{R}^{n}$.

Step I. Prove that $W^{\varepsilon}$ is open (equivalently, prove that its complement is closed). To this end, consider a sequence of functions $\left\{w_{k}\right\}_{k}$ such that $w_{k} \notin W^{\varepsilon}$ and $w_{k}$ converges uniformly to $w \in C\left([0,1], \mathbb{R}^{n}\right)$. For each $k \in \mathbb{N}$, there exists $x_{k} \in \mathcal{F}^{w_{k}}$ such that

$$
\int_{[0,1)} \Phi\left(F\left(t, x_{k}(t)\right),\left(x_{k}\right)_{g}^{\prime}(t)\right) d g(t) \geq \varepsilon .
$$

The fact that $\left(x_{k}\right)_{g}^{\prime}(t) \in F\left(t, x_{k}(t)\right) \subset B \mu_{g}$-a.e. means that

$$
\left\|\left(x_{k}\right)_{g}^{\prime}(t)\right\| \leq 1 \quad \text { for } \mu_{g} \text {-a.e. } t \in[0,1] \text { and for all } k \in \mathbb{N} \text {, }
$$


and by Theorem 7 we can find a subsequence of $\left\{x_{k}\right\}_{k}$ (for simplicity, not relabeled) which converges uniformly to a $g$-continuous bounded function $x:[0,1] \rightarrow \mathbb{R}^{n}$. Therefore, for any $s<t$ in $[0,1]$,

$$
\left\|x_{k}(t)-x_{k}(s)\right\| \leq \int_{[s, t)}\left\|\left(x_{k}\right)_{g}^{\prime}(\tau)\right\| d g(\tau) \leq g(t)-g(s), \quad \forall k \in \mathbb{N},
$$

whence $\|x(t)-x(s)\| \leq g(t)-g(s)$, showing that $x \in \mathcal{A C}_{g}\left([0,1], \mathbb{R}^{n}\right)$. On the other hand, the continuity of $F$ implies that for each $t \in[0,1]$ the sequence of sets $F\left(t, x_{k}(t)\right)$ converge in the Pompeiu-Hausdorff distance to $F(t, x(t))$. Consequently $x_{g}^{\prime}(t) \in F(t, x(t)) \mu_{g}$-a.e. and

$$
\begin{aligned}
\left\langle x_{g}^{\prime}(t), w(t)\right\rangle & =\lim _{k \rightarrow \infty}\left\langle\left(x_{k}\right)_{g}^{\prime}(t), w_{k}(t)\right\rangle \\
& =\lim _{k \rightarrow \infty} \max _{z \in F\left(t, x_{k}(t)\right)}\left\langle z, w_{k}(t)\right\rangle=\max _{z \in F(t, x(t))}\langle z, w(t)\rangle \quad \mu_{g} \text {-a.e. }
\end{aligned}
$$

In summary, $x \in \mathcal{F}^{w}$. It remains to show that

$$
\int_{[0,1)} \Phi\left(F(t, x(t)), x_{g}^{\prime}(t)\right) d g(t) \geq \varepsilon
$$

The upper semicontinuity of $\Phi[6$, Proposition 4.2] implies that

$$
\limsup _{k} \Phi\left(F\left(t, x_{k}(t)\right),\left(x_{k}\right)_{g}^{\prime}(t)\right) \leq \Phi\left(F(t, x(t)), x_{g}^{\prime}(t)\right)
$$

Note that by (10), for $\mu_{g}$-a.e. $t \in[0,1]$ and for all $k \in \mathbb{N}$, we have

$$
\int_{0}^{1}\left\|f(s)-\left(x_{k}\right)_{g}^{\prime}(t)\right\|^{2} d s \leq 4
$$

for every $f:[0,1] \rightarrow F\left(t, x_{k}(t)\right)$ such that $\int_{0}^{1} f(s) d s=\left(x_{k}\right)_{g}^{\prime}(t)$. Thus,

$$
\Phi\left(F\left(t, x_{k}(t)\right),\left(x_{k}\right)_{g}^{\prime}(t)\right) \leq 4, \quad k \in \mathbb{N}, \mu_{g} \text {-a.e. } t \in[0,1],
$$

and by the reverse Fatou lemma:

$$
\begin{aligned}
& \underset{k}{\limsup } \int_{[0,1)} \Phi\left(F\left(t, x_{k}(t)\right),\left(x_{k}\right)_{g}^{\prime}(t)\right) d g(t) \\
& \quad \leq \int_{[0,1)} \limsup _{k} \Phi\left(F\left(t, x_{k}(t)\right),\left(x_{k}\right)_{g}^{\prime}(t)\right) d g(t) .
\end{aligned}
$$

This together with (9) and (11) yields

$$
\int_{[0,1)} \Phi\left(F(t, x(t)), x_{g}^{\prime}(t)\right) d g(t) \geq \varepsilon
$$

in other words, $w \notin W^{\varepsilon}$.

Step II. Show that $W^{\varepsilon}$ is dense in $C\left([0,1], \mathbb{R}^{n}\right)$. Since $F\left([0,1] \times \mathbb{R}^{n}\right) \subset B$, we clearly have $F^{w}(t, x)=F^{w /|w|}(t, x), x, w \in \mathbb{R}^{n}, t \in[0,1]$. Recalling that functions in $C\left([0,1], \mathbb{R}^{n}\right)$ can be 
uniformly approximated by continuous functions $w:[0,1] \rightarrow \mathbb{R}^{n} \backslash\{0\}$, we prove the density of $W^{\varepsilon}$ by showing that for an arbitrary continuous function $z:[0,1] \rightarrow \partial B$ and any $\rho>0$, we have

$$
W^{\varepsilon} \cap B(z, 4 \rho) \neq \emptyset,
$$

where $B(z, 4 \rho)$ stands for the open ball in the space $C([0,1], \partial B)$, centered in $z$, with radius $4 \rho$. In other words, we will construct a continuous function $w:[0,1] \rightarrow \partial B$ such that

$$
\begin{aligned}
& \sup _{t \in[0,1]}\|w(t)-z(t)\| \leq 4 \rho, \\
& \int_{[0,1)} \Phi\left(F(t, x(t)), x_{g}^{\prime}(t)\right) d g(t)<\varepsilon
\end{aligned}
$$

for every $x \in \mathcal{A C}_{g}\left([0,1], \mathbb{R}^{n}\right)$ satisfying $x_{g}^{\prime}(t) \in F^{w(t)}(t, x(t)) \mu_{g}$-a.e. in $[0,1]$.

Following Lemma 13(iv), one may choose $\lambda>0$ such that

$$
f_{S(y)} \phi^{\lambda}(t, x, \varsigma) d \varsigma \leq \frac{\varepsilon}{4}, \quad x \in B, y \in \partial B, t \in[0,1]
$$

where $S(y)=\{\varsigma \in \partial B:|\varsigma-y|<\rho\}$. By reasoning like in Step 4 of the proof of [7, Theorem 2], we know that we can choose $v$ points $p_{1}(0), \ldots, p_{v}(0)$ in the spherical cap $S(z(0))$ such that

$$
\left|\frac{1}{v} \sum_{j=1}^{v} h\left(p_{j}(0)\right)-f_{S(z(0))} h(\varsigma) d \varsigma\right| \leq \frac{\varepsilon}{4}
$$

for every $\lambda$-Lipschitz continuous function $h: \mathbb{R}^{n} \rightarrow \mathbb{R}$. Moreover, for each $s \in[0,1]$, the points defined by $p_{j}(s)=\Theta(s) p_{j}(0), j=1, \ldots, v$ (where $\Theta(s)$ is a rotation mapping $z(s)$ to $z(0))$, share the same property. From this it follows that, for each $s \in[0,1]$, we can choose a finite number of points $p_{j}(s) \in S(z(s)$ ), $j=1, \ldots, v$ (with $v$ depending only on $\rho$ ) such that the inequality

$$
\left|\frac{1}{v} \sum_{j=1}^{v} f\left(t, p_{j}(s)\right)-f_{S(z(s))} f(t, \varsigma) d \varsigma\right| \leq \frac{\varepsilon}{4}
$$

holds for any choice of $t \in[0,1]$ and for every function $f:[0,1] \times \mathbb{R}^{n} \rightarrow \mathbb{R}$ such that $f(t, \cdot)$ is $\lambda$-Lipschitz continuous.

Let $g^{C}$ denote the continuous part of $g$. Given $N \in \mathbb{N}$, consider a division $0=s_{0}<s_{1}<$ $\cdots<s_{N}=1$ such that

$$
g^{C}\left(s_{i}\right)-g^{C}\left(s_{i-1}\right)=\frac{g^{C}(1)-g^{C}(0)}{N}, \quad i=1, \ldots, N .
$$

Again by the continuity of $g^{C}$, we can divide each subinterval $\left[s_{i-1}, s_{i}\right]$ into $v$ parts $s_{i-1}=$ $s_{i, 0}<s_{i, 1}<\cdots<s_{i, v}=s_{i}$ such that

$$
g^{C}\left(s_{i, j}\right)-g^{C}\left(s_{i, j-1}\right)=\frac{g^{C}(1)-g^{C}(0)}{N v}, \quad i=1, \ldots, N, j=1, \ldots, v .
$$


Define $z_{N}(s)=p_{j}\left(s_{i}\right)$ for $s \in\left[s_{i, j-1}, s_{i, j}\right), i=1, \ldots, N, j=1, \ldots, v$. Therefore, for $N$ sufficiently large, we have

$$
\sup _{s \in[0,1]}\left\|z_{N}(s)-z(s)\right\|<2 \rho .
$$

Let $x:[0,1] \rightarrow \mathbb{R}^{n}$ be a $g$-Lipschitz continuous function with constant 1 (Definition 5 ). Thus

$$
\begin{aligned}
& \int_{[0,1)} \phi^{\lambda}\left(t, x(t), z_{N}(t)\right) d g^{C}(t) \\
& =\sum_{i=1}^{N} \sum_{j=1}^{v} \int_{\left[s_{i, j-1}, s_{i, j}\right)} \phi^{\lambda}\left(t, x(t), p_{j}\left(s_{i}\right)\right) d g^{C}(t) \\
& \leq \sum_{i=1}^{N} \sum_{j=1}^{v} \int_{\left[s_{i, j-1}, s_{i, j}\right)} \phi^{\lambda}\left(t, x\left(s_{i}\right), p_{j}\left(s_{i}\right)\right) d g^{C}(t) \\
& \quad+\sum_{i=1}^{N} \sum_{j=1}^{v} \int_{\left[s_{i, j-1}, s_{i, j}\right)}\left(\phi^{\lambda}\left(t, x(t), p_{j}\left(s_{i}\right)\right)-\phi^{\lambda}\left(t, x\left(s_{i}\right), p_{j}\left(s_{i}\right)\right)\right) d g^{C}(t) .
\end{aligned}
$$

Noting that for each $t \in[0,1]$ the function $\phi^{\lambda}(t, \cdot, \cdot)$ is $\lambda$-Lipschitz continuous, we get the following estimate:

$$
\begin{aligned}
& \sum_{i=1}^{N} \sum_{j=1}^{v} \int_{\left[s_{i, j-1}, s_{i, j}\right)}\left(\phi^{\lambda}\left(t, x(t), p_{j}\left(s_{i}\right)\right)-\phi^{\lambda}\left(t, x\left(s_{i}\right), p_{j}\left(s_{i}\right)\right)\right) d g^{C}(t) \\
& \quad \leq \sum_{i=1}^{N} \sum_{j=1}^{v} \int_{\left[s_{i, j-1}, s_{i, j}\right)} \lambda\left\|x(t)-x\left(s_{i}\right)\right\| d g^{C}(t) \\
& \quad \leq \sum_{i=1}^{N} \sum_{j=1}^{v} \int_{\left[s_{i, j-1}, s_{i, j}\right)} \lambda\left(g\left(s_{i}\right)-g(t)\right) d g^{C}(t) \\
& \quad \leq \sum_{i=1}^{N} \sum_{j=1}^{v} \lambda\left(g\left(s_{i}\right)-g\left(s_{i-1}\right)\right) \mu_{g}^{C}\left(\left[s_{i, j-1}, s_{i, j}\right)\right) .
\end{aligned}
$$

This together with (14) yields

$$
\begin{aligned}
& \sum_{i=1}^{N} \sum_{j=1}^{v} \int_{\left[s_{i, j-1}, s_{i, j}\right)}\left(\phi^{\lambda}\left(t, x(t), p_{j}\left(s_{i}\right)\right)-\phi^{\lambda}\left(t, x\left(s_{i}\right), p_{j}\left(s_{i}\right)\right)\right) d g^{C}(t) \\
& \quad \leq \lambda \frac{g^{C}(1)-g^{C}(0)}{N} \sum_{i=1}^{N}\left(g\left(s_{i}\right)-g\left(s_{i-1}\right)\right) \\
& \quad=\lambda \frac{g^{C}(1)-g^{C}(0)}{N}(g(1)-g(0)) .
\end{aligned}
$$


On the other hand, for each $i=1, \ldots, N$, applying (13) with the choice $f(t, \varsigma)=$ $\phi^{\lambda}\left(t, x\left(s_{i}\right), \varsigma\right)$ and using (12), we obtain

$$
\sum_{j=1}^{\nu} \phi^{\lambda}\left(t, x\left(s_{i}\right), p_{j}\left(s_{i}\right)\right)<v \frac{\varepsilon}{4}+v f_{S\left(z\left(s_{i}\right)\right)} \phi^{\lambda}\left(t, x\left(s_{i}\right), \varsigma\right) d \varsigma \leq v \frac{\varepsilon}{2} .
$$

Therefore, using (14), the term in (16) can be estimated as follows:

$$
\begin{aligned}
& \sum_{i=1}^{N} \sum_{j=1}^{v} \int_{\left[s_{i, j-1}, s_{i, j}\right)} \phi^{\lambda}\left(t, x\left(s_{i}\right), p_{j}\left(s_{i}\right)\right) d g^{C}(t) \\
& \quad \leq \nu \frac{\varepsilon}{2} \sum_{i=1}^{N} \mu_{g}^{C}\left(\left[s_{i, j-1}, s_{i, j}\right)\right)=\left(g^{C}(1)-g^{C}(0)\right) \frac{\varepsilon}{2} .
\end{aligned}
$$

In summary

$$
\int_{[0,1)} \phi^{\lambda}\left(t, x(t), z_{N}(t)\right) d g^{C}(t) \leq\left(g^{C}(1)-g^{C}(0)\right)\left(\frac{\varepsilon}{2}+\frac{\lambda}{N}(g(1)-g(0))\right) .
$$

Hence, for $N$ sufficiently large, we have

$$
\int_{[0,1)} \phi^{\lambda}\left(t, x(t), z_{N}(t)\right) d g^{C}(t) \leq \frac{3 \varepsilon}{4} .
$$

Having in mind (15), we can choose a continuous function $w:[0,1] \rightarrow \partial B$ such that

$$
\sup _{t \in[0,1]}\|w(t)-z(t)\| \leq 4 \rho \quad \text { and } \quad \mu_{g}^{C}\left(\left\{t \in[0,1] ; w(t) \neq z_{N}(t)\right\}\right)<\frac{\varepsilon}{4} .
$$

By (P), for each $k \in \mathbb{N}, F^{w}\left(t_{k}, x\left(t_{k}\right)\right)$ is a singleton, thus

$$
\phi\left(t_{k}, x\left(t_{k}\right), y\right)=0 \quad \text { for all } y \in \partial B \text {. }
$$

Moreover, $F\left([0,1] \times \mathbb{R}^{n}\right) \subset B$ implies $\phi \leq 1$, and consequently $\phi^{\lambda} \leq 1$. Thus

$$
\begin{aligned}
& \int_{[0,1)} \phi(t, x(t), w(t)) d g(t) \\
& \quad=\int_{[0,1)} \phi(t, x(t), w(t)) d g^{C}(t)+\sum_{k=1}^{\infty} \phi\left(t_{k}, x\left(t_{k}\right), w\left(t_{k}\right)\right)\left(g\left(t_{k}+\right)-g\left(t_{k}\right)\right) \\
& \quad \leq \int_{[0,1)} \phi^{\lambda}(t, x(t), w(t)) d g^{C}(t) \\
& \quad \leq \int_{[0,1)} \phi^{\lambda}\left(t, x(t), z_{N}(t)\right) d g^{C}(t)+\int_{\left\{t ;(t) \neq z_{N}(t)\right\}} \phi^{\lambda}(t, x(t), w(t)) d g^{C}(t)<\varepsilon,
\end{aligned}
$$

that is,

$$
\int_{[0,1)} \phi(t, x(t), w(t)) d g(t)<\varepsilon
$$


for every function $x:[0,1] \rightarrow \mathbb{R}^{n}$ which is $g$-Lipschitz continuous with constant 1 . In particular, the inequality holds for $x \in \mathcal{F}^{w}$ (see Remark 9 and Lemma 6). Hence, recalling the definition of $\phi$, we get

$$
\int_{[0,1)} \Phi\left(F(t, x(t)), x_{g}^{\prime}(t)\right) d g(t) \leq \int_{[0,1)} \phi(t, x(t), w(t)) d g(t)<\varepsilon, \quad x \in \mathcal{F}^{w}
$$

proving that $W^{\varepsilon} \bigcap B(z, 4 \rho) \neq \emptyset$ and, consequently, that $W^{\varepsilon}$ is indeed dense.

Remark 15 Clearly, for $g$ continuous, property (P) plays no role. Furthermore, if $g$ is the identity function, $x_{g}^{\prime}$ coincides with the usual derivative $x^{\prime}$, and consequently Theorem 14 corresponds to a generalization of [7, Theorem 1] to non-autonomous differential inclusions. When dealing with impulsive differential inclusions (5), property $(\mathrm{P})$ is automatically satisfied if, like in [3], the multifunctions $I_{k}$ describing the jumps at the impulse points are single-valued. However, this property allows for more interesting cases of multivalued jumps; for example, if the values of $I_{k}$ are balls or if their boundaries do not contain segments.

According to Theorem 14, the set of continuous functions $w$ satisfying the relation $\mathcal{F}^{w} \subset$ $\mathcal{F}^{\text {ext }}$ is 'large' enough. Recalling that Theorem 11 ensures that $\mathcal{F}^{w}$ is non-empty for any choice of $w \in C\left([0,1], \mathbb{R}^{n}\right)$, we conclude the following.

Theorem 16 Let $g:[0,1] \rightarrow \mathbb{R}$ be a non-decreasing left-continuous function and $F:$ $[0,1] \times \mathbb{R}^{n} \rightarrow \mathcal{P}_{\text {ck }}\left(\mathbb{R}^{n}\right)$ be a bounded Pompeiu-Hausdorff continuous multifunction with property (P). Then the g-differential inclusion (6) admits at least one solution.

Combining the theorem above with the correspondence described in Theorem 10, we get the following result regarding extremal solutions for measure differential inclusions.

Corollary 17 Let $\mu$ be a finite regular Borel measure and $F:[0,1] \times \mathbb{R}^{n} \rightarrow \mathcal{P}_{\mathrm{ck}}\left(\mathbb{R}^{n}\right)$ be a bounded Pompeiu-Hausdorff continuous multifunction such that the set $F^{w}(t, x)$ is a singleton for every $w, x \in \mathbb{R}^{n}$, whenever $\{t\} \subset[0,1]$ is an atom of $\mu$. Then the problem

$$
d x(t) \in F(t, x(t)) d \mu, \quad x(0)=0,
$$

admits extremal solutions.

Another important consequence of Theorem 14 is an existence result for non-convex valued $g$-differential inclusions generalizing the classical result in [19].

Theorem 18 Let $g:[0,1] \rightarrow \mathbb{R}$ be a non-decreasing left-continuous function and $G:$ $[0,1] \times \mathbb{R}^{n} \rightarrow 2^{\mathbb{R}^{n}}$ be a bounded multifunction with compact values, continuous with respect to the Pompeiu-Hausdorff distance and such that, for each discontinuity point $t$ of $g$ and for every $x \in \mathbb{R}^{n}$, the set $G(t, x)$ is convex and satisfies $(\mathrm{P})$. Then the problem

$$
x_{g}^{\prime}(t) \in G(t, x(t)) \quad \mu_{g} \text {-a.e., } \quad x(0)=0,
$$

admits at least one solution. 
Proof Define $F:[0,1] \times \mathbb{R}^{n} \rightarrow \mathcal{P}_{\text {ck }}\left(\mathbb{R}^{n}\right)$ by

$$
F(t, x)=\overline{\operatorname{conv}}(G(t, x))
$$

By Mazur's theorem, $F(t, x)$ is, indeed, convex and compact in $\mathbb{R}^{n}$. Furthermore, the hypothesis over $G$ ensures that $F$ has property (P). Therefore, by Theorem 14, the problem

$$
x_{g}^{\prime}(t) \in \operatorname{ext} F(t, x(t)) \quad \mu_{g} \text {-a.e., } \quad x(0)=0
$$

admits a solution. Recalling that compact sets contain the extreme points of their closed convex hulls (Krein-Millman theorem), the result follows.

Thanks to the relation previously described between $g$-differential inclusions and differential inclusions driven by measures, we then obtain the following Filippov-type result for measure differential inclusions.

Corollary 19 Let $\mu$ be a finite regular Borel measure and $G:[0,1] \times \mathbb{R}^{n} \rightarrow 2^{\mathbb{R}^{n}}$ be a bounded multifunction with compact values, continuous with respect to the PompeiuHausdorff distance and such that, when $\{t\} \subset[0,1]$ is an atom of $\mu$, we have $G(t, x)$ convex and for every $w, x \in \mathbb{R}^{n}$, the set $G^{w}(t, x)$ is a singleton. Then the problem

$$
d x(t) \in G(t, x(t)) d \mu, \quad x(0)=0,
$$

admits at least one solution.

\section{Acknowledgements}

The authors are deeply indebted to the anonymous reviewers for their valuable suggestions

\section{Funding}

The research of G. Monteiro has been supported by RVO: 67985840. The second author has been supported by "Excellence in Advanced Research, Leadership in Innovation and Patenting for University and Regional Development"—EXCALIBUR, Grant Contract no. 18PFE / 10.16.2018 Institutional Development Project—Funding for Excellence in RDI, Program 1-Development of the National R \& D System, Subprogram 1.2-Institutional Performance, National Plan for Research and Development and Innovation for the period 2015-2020 (PNCDI III).

\section{Competing interests}

The authors declare that they have no competing interests.

\section{Authors' contributions}

All authors contributed equally and significantly in writing this paper. All authors read and approved the final manuscript.

\section{Author details}

${ }^{1}$ Institute of Mathematics of the Czech Academy of Sciences, Prague, Czech Republic. ${ }^{2}$ Faculty of Electrical Engineering and Computer Science; Integrated Center for Research, Development and Innovation in Advanced Materials, Nanotechnologies, and Distributed Systems for Fabrication and Control (MANSiD), Stefan cel Mare University of Suceava, Suceava, Romania.

\section{Publisher's Note}

Springer Nature remains neutral with regard to jurisdictional claims in published maps and institutional affiliations.

Received: 28 February 2019 Accepted: 4 June 2019 Published online: 20 June 2019

\section{References}

1. Abada, N., Agarwal, R.P., Benchohra, M., Hammouche, H.: Impulsive semilinear neutral functional differential inclusions with multivalued jumps. Appl. Math. 56, 227-250 (2011)

2. Aubin, J.P., Cellina, A.: Differential Inclusions. Springer, Berlin (1984) 
3. Benchohra, M., Henderson, J., Ntouyas, S.: Impulsive Differential Equations and Inclusions. Hindwi Publishing Corporation, New York (2006)

4. Bendová, H., Malý, J.: An elementary way to introduce a Perron-like integral. Ann. Acad. Sci. Fenn., Math. 36(1), 153-164 (2011)

5. Bohner, M., Peterson, A.: Dynamic Equations on Time Scales: An Introduction with Applications. Birkhäuser, Boston (2001)

6. Bressan, A.: The most likely path of a differential inclusion. J. Differ. Equ. 88, 155-174 (1990)

7. Bressan, A.: Extremal solutions of differential inclusions via Baire category: a dual approach. J. Differ. Equ. 255 2392-2399 (2013)

8. Bressan, A., Staicu, V.: Random extremal solutions of differential inclusions. Nonlinear Differ. Equ. Appl. (2016). https://doi.org/10.1007/s00030-016-0375-0

9. Bruckner, A.M., Bruckner, J.B., Thomson, B.S.: Real Analysis. Prentice-Hall, Upper Saddle River (1997)

10. Cao, Y., Sun, J.: On existence of nonlinear measure driven equations involving non-absolutely convergent integrals. Nonlinear Anal. Hybrid Syst. 20, 72-81 (2016)

11. Cichoń, M., Satco, B.: Measure differential inclusions-between continuous and discrete. Adv. Differ. Equ. (2014). https://doi.org/10.1186/1687-1847-2014-56

12. Cichoń, M., Satco, B.: On the properties of solutions set for measure driven differential inclusions. Discrete Contin. Dyn. Syst. 2015 (Special Issue), 287-296 (2015)

13. Cichoń, M., Satco, B., Sikorska-Nowak, A.: Impulsive nonlocal differential equations through differential equations on time scales. Appl. Math. Comput. 218, 2449-2458 (2011)

14. Daniell, P.J.: Differentiation with respect to a function of limited variation. Trans. Am. Math. Soc. 19(4), 353-362 (1918)

15. De Blasi, F.S., Pianigiani, G.: Baire's category and relaxation problems for locally Lipschitzian differential inclusions on finite and infinite time intervals. Nonlinear Anal. 72, 288-301 (2010)

16. Di Piazza, L., Marraffa, V., Satco, B.: Closure properties for integral problems driven by regulated functions via convergence results. J. Math. Anal. Appl. 466, 690-710 (2018)

17. Federson, M., Mesquita, J.G., Slavík, A.: Measure functional differential equations and functional dynamic equations on time scales. J. Differ. Equ. 252, 3816-3847 (2012)

18. Federson, M., Mesquita, J.G., Slavík, A.: Basic results for functional differential and dynamic equations involving impulses. Math. Nachr. 286, 181-204 (2013)

19. Filippov, A.F.: The existence of solutions of multivalued differential equations. Mat. Zametki 10, 307-313 (1971)

20. Frigon, M., López Pouso, R.: Theory and applications of first-order systems of Stieltjes differential equations. Adv. Nonlinear Anal. 6, 13-36 (2017)

21. Kurzweil, J.: Generalized ordinary differential equations and continuous dependence on a parameter. Czechoslov. Math. J. 7, 418-449 (1957)

22. López Pouso, R., Márquez Albés, I.: General existence principles for Stieltjes differential equations with applications to mathematical biology. J. Differ. Equ. 264, 5388-5407 (2018)

23. López Pouso, R., Márquez Albés, I., Monteiro, G.A.: Extremal solutions of systems of measure differential equations and applications in the study of Stieltjes differential problems. Electron. J. Qual. Theory Differ. Equ. (2018). https://doi.org/10.14232/ejqtde.2018.1.38

24. López Pouso, R., Rodriguez, A.: A new unification of continuous, discrete, and impulsive calculus through Stieltjes derivatives. Real Anal. Exch. 40, 319-353 (2015)

25. Monteiro, G.A., Satco, B.: Distributional differential and integral problems: equivalence and existence results. Electron. J. Qual. Theory Differ. Equ. (2017). https://doi.org/10.14232/ejqtde.2017.1.7

26. Monteiro, G.A., Slavík, A., Tvrdý, M.: Kurzweil-Stieltjes Integral: Theory and Applications. World Scientific, Singapore (2019)

27. Satco, B.: Measure integral inclusions with fast oscillating data. Electron. J. Differ. Equ. 2015, 107 (2015)

28. Schwabik, Š.: Generalized Ordinary Differential Equations. World Scientific, Singapore (1992)

29. Silva, G.N., Vinter, R.B.: Measure driven differential inclusions. J. Math. Anal. Appl. 202, 727-746 (1996)

30. Slavík, A.: Dynamic equations on time scales and generalized ordinary differential equations. J. Math. Anal. Appl. 385 534-550 (2012)

31. Stewart, D.E.: Reformulations of measure differential inclusions and their closed graph property. J. Differ. Equ. 175(1) 108-129 (2001)

32. Tolstonogov, A.A.: Differential Inclusions in a Banach Space. Kluwer, Dordrecht (2000)

33. Ward, A.J.: The Perron-Stieltjes integral. Math. Z. 41, 578-604 (1936)

34. Young, W.H.: On integrals and derivatives with respect to a function. Proc. Lond. Math. Soc. 15 (1), 35-63 (1917) 